\title{
Evolutionary Algorithms in Crystal Structure Analysis
}

\author{
Attilio Immirzi ${ }^{1}$, Consiglia Tedesco $^{2}$ and Loredana Erra ${ }^{3}$ \\ ${ }^{1,2}$ Dept. of Chemistry, University of Salerno \\ ${ }^{3}$ European Synchrotron Radiation Facility \\ ${ }^{1,2}$ Italy \\ ${ }^{3}$ France
}

\section{Introduction}

Evolutionary algorithms (EA, see for a general introduction Holland, 1975; Goldberg, 1989; Davis, 1991; Back, 1996) are "probabilistic" optimization procedures. Differently from the "deterministic" ones (yielding an unique and reproducibile solution for assigned input data), EA are somewhat aleatory and not reproducible. Such methods are part of the heuristics (Polya, 1971). To be pedantic the term algorithm should indicate some sequence of mathematical operations producing a foreseeable result if applied to definite input data. There is a resounding oxymoron in the terms evolutionary algorithm. This contradictory language is however diffuse; as this one is not an erudite essay of epistemology, we prefer to be tolerant and fly over on semantic questions. Really evolutionary methods have aleatory routes and also aleatory conclusions in many cases (e.g. stock exchange estimate, weather forecast, etc.); in some cases instead (it happens in crystallography), unique and certain results can be reached in spite of the multiplicity of routes travelled.

Multi-parameter optimization problems are encountered in many fields: industrial process planning, financial investments, environment control, hurricane evolution, and many others (Weise, 2009). We shall however not consider the general aspects of EA but rather to treat about a specific argument: the study of chemical structures based on X-ray diffraction. In this field, as shown later, there is a lot of a priori and useful information so that the aleatority of the procedure can be considerably attenuated. Evolutionary methods in crystallography are better formulated as Constraint Satisfaction Problems (CSP, see Ionita et al., 2010).

Evolutionary methods have a future in crystallography, particularly in solving hard problems. The structural elucidation from X-ray powder diffraction data is among these (section 4.1). However, procedures are not consolidated yet, there are numerous alternatives to be considered for which the common sense and the experience, rather than the theory, could have a role.

This article will not treat the EA in the crystallographic context in its generality; rather it will summarize some ideas and strategies which yielded a specific procedure: it is a possible route, not the only possible one. These procedures are now included in a general-purpose computer program with numerous options for finding and refining crystal structures termed TRY (Immirzi, 2007b). In its actual structure the EA procedure needs that many parameters 
are assigned by the user. Assigning them in a more automatic fashion could no doubt improve the program versatility.

The effectiveness of EA in solving crystal structures by diffraction methods is improved if one makes systematically use of the so called internal coordinates (i.c.) for describing structures, instead of the more common cartesian coordinates. The i.c. were first considered, in diffraction analysis, by Arnott \& Wonacott (1966). A computer program performing least-square refinement of i.c. (LALS, Linked Atom Least Square) was first issued (Smith \& Arnott, 1978) and later updated repeatedly (the last edition, WINLALS , was published by Okada et al., 2003). LALS has been used mainly in studying polymers. Also Tadokoro (1975) discussed the use of i.c. in polymer case.

Both Arnott (and disciples) and Tadokoro did however neglect a very important complication: the mutual independency (non-redundancy) of i.c., being of great importance in all crystallogaphic applications, and in particular in EA, as will be shown later.

Now a procedure for carrying-out a molecular building always using non-redundant i.c. has been devised (Immirzi 2007a,b); it is very simple, practical, and perfectly analytical, runs thoroughly, and eliminates the necessity of using exotic instruments in matrix manipulations (pseudoinversion, diagonalization, etc.; note that the mathematic instruments employed are those of Newton, Gauss, and Lagrange).

\section{Crystalline matter and X-ray diffraction}

Characterization of chemicals, both natural and synthetic, is done always looking at the matter at the atomic scale. In $19^{\text {th }}$ century, atoms were speculative entities and scientists had not instruments for ascertaining their shape or position. Nevertheless, in the middle of century, some of them (Kekulé, Le Bel, van't Hoff and others), with evident attitude versus the heuristics (without awareness of course as heuristics did not exist yet) were persuaded about molecules (proposed many years before by Avogadro finding much skepticism); they begun, with much imagination and using logical arguments, to give them definite shapes, for instance the tetrahedral shape to methane and the hexagonal shape to benzene. Many of these intuitions were demostrated later be perfectly exact. Today to ascertain the geometrical structure of molecules is not more a work of imagination: it is an exact science. Structure is a "property" of any pure substance, defined and reproducible, like colour, density, melting temperature, etc. ${ }^{1}$

The discovery of X-ray diffraction by crystals by Laue in 1912 made possible to ascertain the structure. In the crystalline state, necessarily solid, atoms are ordered: there is a small portion of space, a parallelepiped, generally oblique, termed the unit cell, which repeats itself by translation in three directions and generates the whole solid. If the disposition of atoms in the cell is determined, the whole crystal is determined. Frequently there is also symmetry: only a fraction of the unit cell is independent; the remainder if given by appropriate orthogonal transforms. Edges and interaxial angles of the unit cell are collectively termed lattice constants. Just order makes possible X-ray diffraction of crystal, whose study permits to guess the matter at the atomic scale; resolution is of the order of $0.02 \AA$ or better; consider that the separation of bonded atoms is $1-2 \AA$. The size of molecules studied by X-ray diffraction was initially modest; now studying 50-100 atom molecules is a common matter.

\footnotetext{
${ }^{1}$ There are indeed exceptions: some substances exhibit more than one structure (polymorphism). Crystalline minerals are frequently polymorphic.
} 


\section{Molecules and molecular crystals}

Molecules are finite assemblies of atoms joined by strong forces (chemical bonds). In molecular crystals molecules are orderly assembled through well weaker non-bonding forces (van der Waals forces, dipole-dipole forces, hydrogen bonds, etc.). ${ }^{2}$

Thanks to the repetition, crystals diffract $X$-ray radiation: when a beam of monochromatic radiation strikes a crystalline sample many beams emerge and their intensities can be one-by-one measured. The intensity of the diffracted beams is related to the molecular structure (see below). ${ }^{3}$

In absence of symmetry, if $V$ is the volume of the unit cell, $\lambda$ the radiation wavelength, and $2 \vartheta_{m}$ the highest angular deviation, there are altogether $3.35\left(V / \lambda^{3}\right) \sin ^{3} \vartheta_{m}$ different diffracted beams (reflections). The above relationship indicates that the number of reflections raises as $\sin \vartheta_{m} / \lambda$ increases. The latter is an important parameter: the higher is $\sin \vartheta_{m} / \lambda$ the higher is the chance of success in a structural analysis, and the higher is the accuracy of the result. Consider however that the intensity of the reflections decreases as $\vartheta$ angle increases and that $\sin \vartheta_{m} / \lambda$ may be limited by natural circumstances: there are materials which are intrinsecally weakly diffracting; their structural study cannot be done accurately.

\section{Structure and diffraction}

It is possible to obtain crystal structures from X-ray diffraction data thanks to the mathematical relationship between atomic positions and the intensities of the reflections. The latter, as numerous as the volume of the unit cell is large (see above), are singled out by three integer numbers: the Bragg indices $(h, k, \ell)$. Each is deviated from the incident direction by a characteristic angle $2 \vartheta$, an analytical function of the Bragg indices and of the lattice constants. One must intercept these beams and measure intensity.

There are nowadays very sophisticated instruments (diffractometers), controlled by computers, allowing, with a minimal human intervention and in a short time, a complete characterization of a crystal: measurement of the lattice constants, diagnosis of symmetry, localization and measurement of all reflections with their Bragg indices $h, k, \ell$. If the unit cell contains $N$ atoms, and $x_{j}, y_{j}, z_{j}$ are the atomic coordinates (referred to the unit cell), the complex quantities

$$
F(h, k, \ell)=\sum_{j=1}^{N} f_{j} \exp \left[2 \pi i\left(h \frac{x_{j}}{a}+k \frac{y_{j}}{b}+\ell \frac{z_{j}}{c}\right)\right]
$$

are termed structure factors. The $f_{j}$ (atomic factors) are known real quantities which depend on the chemical nature of the $j$-th atom and on $\sin \vartheta / \lambda$. The larger is the atomic number the higher are $f_{j}$. In every cases $f_{j}$ decreases as $\sin \vartheta / \lambda$ increases.

The above relationship applies at $0 \mathrm{~K}$; at higher temperature $F^{\prime}$ s are somewhat reduced because of the thermal vibration. There are of course as many $F(h, k, \ell)$ as reflections.

Now the squared moduli of $F(h, k, \ell)$ should be orderly proportional to the measured diffraction intensities $I(h, k, \ell)$. This occurs, of course, when the $x_{j}, y_{j}, z_{j}$ coordinates are the true ones. To get the unknown crystal structure one must find all the $x_{j}, y_{j}, z_{j}$ rendering the

\footnotetext{
${ }^{2}$ Not all crystalline materials are molecular. There also ionic crystals and extended covalent structures.

3 The deviation of diffracted beams from the incident direction obeys to the Bragg's law. Geometry is quite similar to the case of a reflecting mirror: diffracted beams can be considered as "reflected" by the sample. For this reason they are frequently termed reflections
} 
$|F(h, k, \ell)|^{2}$ as close as possible to the $I(h, k, \ell)$. Introducing the residual

$$
\chi^{2}=\sum_{h k \ell}\left[I(h, k, \ell)-|F(h, k, \ell)|^{2}\right]^{2}
$$

one can assume that the solution is the one rendering $\chi^{2}$ a minimum. For this reason to find a structure is to solve a global optimization problem. The above $\chi^{2}$, divided by the sum of $F^{2}(h, k, \ell)$ is the common $R_{2}$ index, widely used as fitness function.

\subsection{Single-crystals and polycrystalline samples}

X-ray diffraction studies use either single-crystal samples or polycrystalline samples (powder, fiber). In the second case things are more difficult because all diffracted beams having a given $2 \vartheta$ are overlapped. While the diffraction pattern of a single crystal is a three-dimensional function $I(h, k, \ell)$ rich of information, the powder diffraction is an uni-dimensional function $I(2 \vartheta)$ with poor information.

Structural studies based on powder diffraction data meet the difficulty of measuring the diffracted intensities one-by-one, singled out by the Bragg indices, in presence of overlap. The difficulty can be overcome employing deconvolution techniques (see e.g. Harris, 1998) or, alternatively, renouncing to the separation of reflections and considering the quasi-continous function $I(2 \vartheta)$ (Rietveld, 1967; Rietveld, 1969; Young, 1995) exploiting the so called full-pattern powder profile analysys.

In this case, the measured diffraction intensities $I\left(2 \vartheta_{i}\right)$ are compared with the computed ones given by

$$
I_{c a l c d, i}=\sum_{k} F_{k}^{2} \Omega\left(2 \vartheta_{i}-2 \vartheta_{k}\right)
$$

where the sum is extended to all the reflections whose Bragg $2 \vartheta_{k}$ fall near to the current $2 \vartheta_{i}$ point. In equation (3) $\Omega\left(2 \vartheta_{i}-2 \vartheta_{k}\right)$ is the peak function having a maximum for $2 \vartheta_{i}=2 \vartheta_{k}$. In full-pattern profile analysis the residual $\chi^{2}$ considered is given by the expression

$$
\chi^{2}=\sum_{2 \vartheta_{i}}\left[I\left(2 \vartheta_{i}\right)-I_{c a l c d, i}\right]^{2}
$$

and the most used fitness index is $R_{w p}=\chi^{2} / \sum I^{2}(2 \vartheta)$

Just because EA techniques need a reduced number of data they are attractive in studies based on powder diffraction, particularly when the full-pattern profile analysis is performed. For recent studies see Harris (1998), Feng (2006), Hanson (2007), Oganov (2006).

When a structure has been solved (at a coarse level) a second problem arises: to refine atomic positions. This is a local optimization problem. Once again the goal is to find the minimum of $\chi^{2}$ varying systematically atomic positions in the vicinity of the initial coarse values and adding some parameters for expressing the thermal vibration.

While the local optimization is done using deterministic algorithms (typically a least-square refinement) and proceeds, in most cases, without problems, the global optimizazion, even at coarse level, is much more exacting.

The remainder of this chapter concerns only global optimization problems. In addition we shall not treat the traditional solution methods but only some based on evolutionary algorithms. 


\section{The three-dimensional space and the problem space}

Solving a structure of $N$ atoms means finding $N$ positions in the $3 \mathrm{D}$ space. Unfortunately there are no algorithms for finding positions one by one (unless rather simple cases occurring in inorganic chemistry). Atomic positions must be find simultaneously.

An alternative way to define the terms of problem is to refer to another space, the so called problem space, having $3 \mathrm{~N}$ dimensions instead of three. To catch a structure means to locate a single point in this hyper-space. Simple to say, but very difficult to do. As explained later the EA follow this crazy idea.

Small-medium size problems, are solved using deterministic algorithms, provided that the available diffraction measurements are numerous, accurate, and complete (i.e. including all the intensities with the diffraction angles $\vartheta$ lower than the assigned $\left.2 \vartheta_{m}\right)$. For these methods, not discussed in this article, there are many excellent textbooks (e.g. Giacovazzo et al., 2002; Stout \& Jensen, 1995). ${ }^{4} \quad$ The EA are indicated instead in difficult cases: limited $2 \vartheta_{m}$, poor quality of data, low number of reflections.

One could ingenuously believe that all structural problems can be solved moving the representative point systematically in the whole problem-space and examining all solutions. Atomic coordinates are however real and continuous quantities; so points are infinite. Well, even though finite (and coarse) intervals are considered to render the problem-space discontinuous, one obtains astronomic numbers also in the simple cases (see later). The systematic exploration of the whole problem-space is not a practical tool, unless some tricks are adopted. Possible strategies are:

i) to reduce substantially the number of variables adopting other kinds of coordinates rather than crystallographic (see section 6) and choosing coordinate systems for which a good fraction of variables are predictable;

ii) to render the problem-space discontinuous;

iii) to reduce as much as possible the range of variation for each variable.

We shall suppose that the crystal under study is a molecular crystal with known composition (chemical formula) and known interatomic connection (structural formula); consider that the chemical formula is obtained by chemical analysis; structural formula is the result of a number of physical observations (e.g. NMR, IR, UV spectroscopy) and chemical observations (relations of the unknown substance to other already known).

Using diffraction techniques one can establish size, shape, and symmetry of the unit cell. From the experimental density of crystals one obtains the atom content of the unit cell. Using the diffractometers one obtains an appropriate number of diffracted intensities.

\section{Coordinates}

Structures are commonly described by using the crystallographic coordinates (c.c.) to define atom positions. The c.c. (collectively indicated with $p_{i}$ ) are $3 N$ if atoms are $N$. This choice is the most natural and also practical because the simple analytical relationship with the structure factors $F(h, k, \ell)$, see equation (1).

\footnotetext{
${ }^{4}$ Among deterministic methods we include the so called direct methods, actually the most used. Also direct methods are somewhat probabilistc and aleatory, in modest amount however. Also using direct methods the risk of finding false solutions exists.
} 
As a matter of fact, infinite alternative systems for describing structures are conceivable: if new coordinates $q_{i}$ are introduced, so that one can transform from $p_{i}$ to $q_{i}$ and viceversa using analytic and biunivocal relationships, the $q_{i}$ can be used as alternative stuctural variables. The number of $q_{i}$ must be, of course, again $3 N .^{5}$

Which are the alternative useful descriptions among the infinite possible ones? To reply it is important to point out that atomic positions are not fully impredictable. The systematic study of molecular crystals did demonstrate that atomic positions are not random quantities. Any structural hypothesis must obey to the following rules:

i) atoms must be appropriately separated among them; for each atom pair distance is anything but random: if the two atoms are chemically bound, atom separation is a bond-length (b-l) and must be close to the sum of the atomic radii (see e.g. Cotton, 1999); if not bound and spaced by three bonds or more, the distance must be higher than the sum of the so called van der Waals radii (Bondi, 1964); the same applies when the two atoms belong to different molecules (packing distances).

ii) the bond-angles (b-a) must be close to the "canonical" values prescribed by the rules of orbital hybridation (see e.g. Cotton, 1999): angles close to $109.5^{\circ}$ on carbon atoms with $s p^{3}$ hybridation, close to $120^{\circ}$ on carbon atoms with $s p^{2}$ hybridation, etc. The examination of "molecular models" suggest in much cases angles in rather restricted intervals.

iii) the molecular conformation must obey to the rules of stereochemistry: aromatic rings must have $D_{6 d}$ symmetry, the sequences $\mathrm{C}-\mathrm{C}=\mathrm{C}-\mathrm{C}$ have to be planar, the torsion-angles $(\mathrm{t}-\mathrm{a})$ about single bonds must have values close to those typical of ethane-like molecules $\left(-60,60,180^{\circ}\right)$, etc. These limitations can be thus "rigid" in some cases (double bonds), "flexible" in other cases (single bonds).

The above restrictions for $b-1, b-a$, and $t-a$ are of simple mathematical formulation if the crystallographic coordinates $p_{i}$ are employed; there is however the disadvantage that the restrictions (constraints) apply not to the $p_{i}$ themselves, but to a number of mathematical functions of the $p_{i}$. Indeed there are procedures for doing minimizations in presence of constraints: the Lagrange method (Goldstein, 1980) with the drawback that only a few constraints can be accounted for, while in molecular building there are numerous. Instead, introducing alternative coordinates $q_{i}$ chosen with cleverness, it is possible to impose the above constraints not on the functions but directly on the $q_{i}$. If so happens one simply removes the $q_{i}$ from the list of variables.

\section{The internal coordinates. Eyring algorithm}

It is customary studying complicate problem by means of the so called internal coordinates (i.c.), used in performing molecular building in various other contexts (e.g. spectroscopy, theoretical chemistry). The pioneer work (in chemistry) was that of Eyring (1932) who devised the procedure later called Z-matrix . Really the idea is well older; it was skilfully described by Lagrange (1796) who coined the term generalized coordinates. Lagrange studied not molecules but machines; things are rather similar after all: Lagrange's machines are made by rigid objects

\footnotetext{
${ }^{5} 3 \mathrm{~N}$ coordinates are necessary for describing the crystal structure. If only the molecular structure is of interest, the coordinates are $3 N-6$ being six the rigid body coordinates (Goldstein 1980).
} 
connected to each other with limited freedom of movement; in molecules the rigid objects are the chemical bonds and movements are limited to torsion and (in small amount) to bending. ${ }^{6}$ Eyring's algorithm is simple, provided that there are neither rings nor polyhedra. The idea is to build molecules first defining three atoms, ( $\mathrm{C} 1, \mathrm{C} 2$, and $\mathrm{C} 3$ in Fig. 1) then inserting the other atoms stepwise as function of three i.c. in each step: a b-l $b, a \mathrm{~b}-\mathrm{a} \tau$ and a $\mathrm{t}-\mathrm{a} \vartheta$. Of course, in each step one must specify which atoms are to be considered.

Three i.c. are needed for starting a molecular building, viz. two b-l $\left(b_{1}, b_{2}\right)$ and a b-a $\left(\tau_{3}\right)$. It is customary to put the 1st atom on the origin, the 2 nd atom along $x$ axis, the 3rd atom in the $x, y$ plane with $y>0$.

Fig. 1 shows how the building starts (chbe command, green atoms) and the Eyring construction (red atom). The construction of the 5th atom (blue) is discussed later.

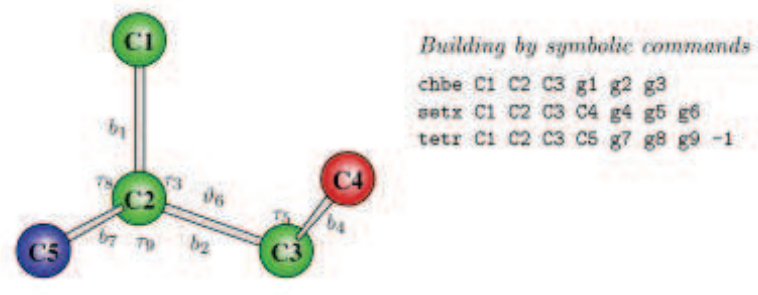

Fig. 1. Building a 5 atoms molecule

The Eyring's machinery for attaching a new atom after a given sequence A, B, C, is as follows: one computes the vector $\mathbf{v}$ with components $b \cos \tau, b \sin \tau \cos \vartheta, b \sin \tau \sin \vartheta$, the vector product $\mathbf{u}=(B-A) \times(C-B)$, and the orthogonal transformation matrix $\mathbf{T}$ aligning $\mathrm{A}$ and $\mathrm{B}$ points along $z^{\prime}$ and making $y_{C}^{\prime}=x_{C}^{\prime}=0$; then adds to $C$ atom the product $\mathbf{T} \cdot \mathbf{v}$. The analytical expression of $\mathbf{T}$ matrix is:

$$
\mathbf{T}=\left|\begin{array}{lll}
x_{B}-x_{A} & x_{C}-x_{B} & u_{x} \\
y_{B}-y_{A} & y_{C}-y_{B} & u_{y} \\
z_{B}-z_{A} & z_{C}-z_{B} & u_{z}
\end{array}\right|
$$

with each column normalized to length 1 .

Besides the Eyring's construction ( $\operatorname{set} \mathrm{x}$ ) needing three variables $b, \tau, \vartheta$, another construction is practical when atoms are inserted on tertiary carbon atoms of known chirality; this construction (tet $r$ ) makes use of two b-a $\tau_{1}, \tau_{2}$ (instead of one b-a and one $t-a$ ); the advantage is the restricted range of the b-a compared with the wide range of the $t$-a. The above 5 -atom skeleton (Fig. 1) has been built just with 3 constructions: chbe (for atoms $\mathrm{C} 1-\mathrm{C} 2-\mathrm{C} 3$, in green), setx (for atom C4, in red), and tetr (for atom C5, in blue).

In Fig. 1 the three constructions are indicated "symbolically" according to a conventional syntax (see later).

Of course six other variables must be added to the molecular i.c. for defining the actual position and orientation of the molecule in the unit cell: three translations and three rotation angles. Rototranslation i.c. can be less than six because of crystal symmetry.

\footnotetext{
${ }^{6}$ This representation applies when bonds are considered rigid and bond-angles semirigid. This situation applies in studying molecular crystals (at a coarse level). In other contexts (e.g. spectroscopy) things are different.
} 


\subsection{Redundant coordinates}

A serious drawback encountered in building molecules using Eyring method is the possible redundancy of the i.c. In simpler words they might be not independent. While independent i.c. can be varied each in turn preserving the stuctural formula, this does not apply in case of redundancy. When molecular building is performed within random search and genetic procedures, the preservation of the structural formula is of basic importance. Also regarding reduncancy there is nothing new after Lagrange; he said all the necessary: the generalized coordinates are exactly the same thing.

As illustrated below, non-redundancy in molecular building can be fulfilled by selecting the $3 N-6$ i.c. of a molecule with shrewdness among $\mathrm{b}-\mathrm{l}, \mathrm{b}-\mathrm{a}, \mathrm{t}-\mathrm{a}$, and also bending angles if necessary (see later). Bending angles become necessary in two cases: cyclic molecules and polyhedric molecules.

\subsection{Cyclic molecules}

A possible solution overcoming redundancy in cyclic molecules is shown in Fig. 2 considering the case of cyclohexane.

Since the $g_{i}$ must be, at a molecular level, $3 N-6$, considering $N$ bond-lengths, only other $2 N-6 g_{i}$ must be assigned, necessarily, angular. The method adopted (Immirzi, 2007a,b) integrates Eyring's procedure for building atoms C1, C2, .. C5 (9 $g_{i}$ are employed altogether, $4 \mathrm{~b}-\mathrm{l}, 3 \mathrm{~b}-\mathrm{a}$, and $2 \mathrm{t}-\mathrm{a}$ ) with a new machinery for building atom C6 (proposed by Goto and Osawa, 1989) using two b-l and one angle only, a so called bending angle $(\varphi)$; with this construction the molecule is bent about a line crossing two atoms separated by one bond only. Such construction (termed $f l a p$ ) has been added to the above ones. The machinery for computing $\mathrm{C} 6$ as a function of $\varphi$ and the two b-1 C1-C6 $\left(g_{11}\right)$ and C5-C6 $\left(g_{12}\right)$ is very simple (see Immirzi, 2005a).

The construction shown in Fig. 2, termed flap resolves the problem for rings of any dimension and also every polyciclic molecule (e.g. decaline, steroids, etc.) and resolves also intricate multicyclic molecules like norbornane, pinene, spirocompounds, etc.

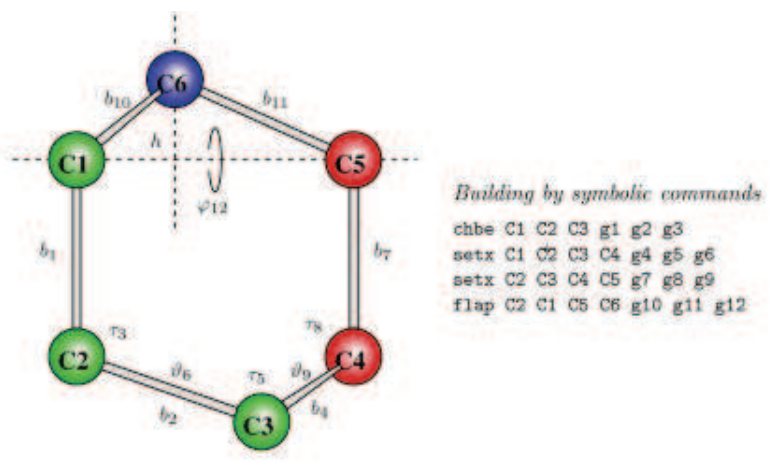

Fig. 2. Building a ring without redundancy, cyclohexane $\mathrm{C}_{6} \mathrm{H}_{6}$, (the blue atom is computed by a flap instruction).

\subsection{Polyhedric molecules}

A possible solution overcoming redundancy in polyhedric molecules is shown in Fig. 3 considering the simplest polyhedron: the cubane $\mathrm{C}_{8} \mathrm{H}_{8}$, represented as a bare carbon atom 

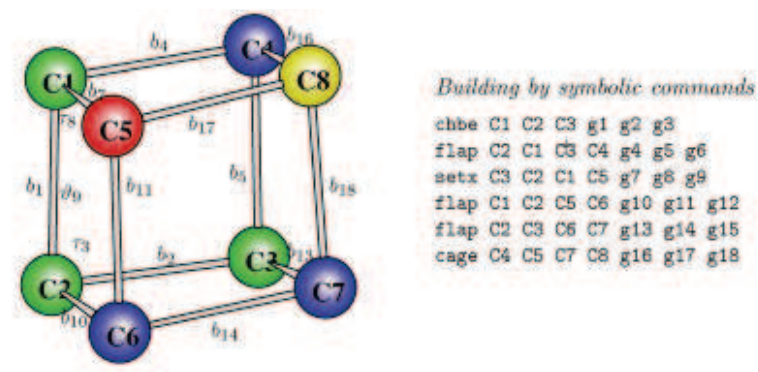

Fig. 3. Building a polyhedric molecule without redundancy (cubane $\mathrm{C}_{8} \mathrm{H}_{8}$ ). The green atoms are computed by chbe, the red atom by set $x$, the blue atoms are computed by flap, and the yellow atom by cage.

skeleton. Remember that the $g_{i}$ must be, at the molecular level, $3 \times 8-6=18$ and $12 g_{i}$ are b-l. In order to build without redundancy one must use only 6 angles. Building the first 7 atoms can be done as indicated symbolically in the figure. After the 5th command one atom is lacking, C8, and all the 6 angles have been used besides 9 over 12 bond-lengths. To complete the molecule one can find $x_{8}, y_{8}, z_{8}$ imposing $\mathrm{C} 8$ has assigned distances $\mathrm{b} 16, \mathrm{~b} 17, \mathrm{~b} 18$ from points C4, C5, C7. This is a simple (and classical) problem of 2nd degree having an unique solution provided that the polyhedron is convex. The command cage computes the lacking atom. Note that cage allows modeling also giant polyhedra like the fullerenes.

\section{Other constructions and symbolic building}

Molecular and crystal building through internal coordinates is not used only in performing structural analysis by EA. It can be used instead in all conventional procedures. In particular for doing interactive modeling, trial-and-error calculation, and at last for doing the least-square refinement of structures based on the i.c. themselves as optimized variables. In the latter case the non-redundancy of the variables plays a crucial role since only in absence of redundancy normal matrices are always non-singular. ${ }^{7}$

For these reasons all the mentioned constructions have been programmed devising an unique subroutine (termed LAGR, an homage to Lagrange, the authentic, and ignored, discoverer of the i.c.) performing the whole construction at each call. To simplify the input data preparation LAGR has been structured so that the various constructions invoked are specified symbolically according to a conventional syntax with one line of data for each construction step. Examples of this syntax are indicated in the figures and in the input data for procedure validation (Tables 1 and 2).

Besides the mentioned four basic constructions needed for building molecules of any kind (chbe, setx, tetr, flap, and cage, tetr is really not indispensable) there are numerous other constructions, not strictly necessary but very useful. Among them there are phen (building phenyl groups), met 1 (building a $\mathrm{CH}_{3}$ group) and others. LAGR subroutine includes also the commands necessary for placing the built molecule in the unit cell, for doing orthogonal transforms, for referring the molecule to its inertial axes, and others. A clever

\footnotetext{
${ }^{7}$ A persuasive argument is that normal matrix needed for carrying out the least-square procedure is computed evaluating the first-order partial derivatives of structure factors vs. the variables. Such derivatives are computed wrong if the variables are not strictly independent to each other.
} 
usage of these commands brings to a further reduction of the effective number of i.c. by imposition of local symmetry, i.e. the identity of structurally independent i.c. but chemically indistinguishable. At last a number of special commands were implemented for the case of linear polymers: to create regular helices and chains with glide-planes, to orient the chain parallel to the crystallographic $c$ edge, etc.

\section{General layout of a EA based procedure. Metaheuristic approach}

The evolutionary (or genetic) algorithms for resolving global optimization problems are based on the idea of finding the "true" solution starting from a number of more or less arbitrary solutions and performing combinations of the variables analogous to the ones occurring in the cellular reproduction.

There is an analogy between chromosomes and molecular structures. As a chromosome can be described by a sequence of genes, a molecular structure can be described by a sequence of structural variables. For these reasons the terms are used as synonyms.

In order to limit the aleatority of the procedure it is practical to follow the so called metaheuristic approach (Weise, 2009) assuming that the $g_{i}$ vary as multiple of a small increments. With this contrivance the i.c. (real and continuous quantities) become integer numbers whose upper limit is limited by the range of excursion of the $g_{i}$ themselves. "Solutions" can binary-coded using a finite number of bits. Consider anyway that the number of points in the problem-space remains astronomic; for instance a 10-variable structure codified in 60-bits may assume $2^{60} \approx 10^{18}$ values. If, for simplicity, a 5-gene case is considered, and 7, 6, 3, 4, 6 bits are dedicated to the 5 genes respectively, the binary code will be (braces groups the bits of given $g$ ):

$$
\underbrace{b_{6} b_{5} b_{4} b_{3} b_{2} b_{1} b_{0}}_{g_{1}} \underbrace{b_{5} b_{4} b_{3} b_{2} b_{1} b_{0}}_{g_{2}} \underbrace{b_{2} b_{1} b_{0}}_{g_{3}} \underbrace{b_{3} b_{2} b_{1} b_{0}}_{g_{4}} \underbrace{b_{5} b_{4} b_{3} b_{2} b_{1} b_{0}}_{g_{5}}
$$

\subsection{Planning the complete structural building}

It is propedeutical to write-up a complete schema for the structural building based on the symbolic language. ${ }^{8} \quad$ This important step is anything but "automatic"; rather it requires much attention and cleverness. One must assign the non-redundant i.c. and prepare the full list of the symbolic building commands which, using the aforementioned subroutine LAGR, will provide the full structural building as function of the current i.c. This schema serves not only for carrying out the EA procedure, but for all the numerous options of TRY program (calculation of structure factor, model adjustment, crystal packing, least-square refinement, geometrical computations, etc.). Among the symbolic instructions there is one indispensable for carrying out the random search in the problem space, termed $x c o n$, and discussed in details later.

\subsection{Binary encoding}

Now the user must: i) decide which $g_{i}$ must be kept fixed and which varied (it is customary to exclude the bond-lengths and, possibly, also the bond-angles); ii) assign to each variable $g_{i}$ a convenient increment $\Delta g_{i}$ (the $g_{i}$ will be varied by multiple of $\Delta g_{i}$ ), iii) assign to each

\footnotetext{
${ }^{8}$ These schemas are familiar to people using the Eyring's mechanism for molecular building. In the present case things are a little bit more difficult for the variety of constructions, which are necessary to avoid redundancy.
} 
variable $g_{i}$ how many bit $\left(m_{i}\right)$ are to be used in binary encoding. $\Delta g_{i}$ and $m_{i}$ determine the range of excursion of the $g_{i}$ (range it is exactly $\Delta g_{i} \times 2^{m_{i}}$ ).

It is evident that some common sense must be exerted in assigning $\Delta g_{i}$ and $m_{i}$ with a compromise between fine steps in exploring the problem-space and wide spanning intervals. For instance, for a bond angle on a carbon atom one could assign $\Delta=1.0^{\circ}$ and $m_{i}=4$; the angle will span a sufficient interval of $16^{\circ}$. Torsion angles (about single bonds), have instead higher uncertainity; giving them e.g. 5 bits and $\Delta g_{i}=3^{\circ}$ will permit a span of $64^{\circ}$. A large number of bits, e.g. 7 or 8 , must be assigned to the molecular rotation angles: using $m=7$ and $\Delta=2.8^{\circ}$ will ensure a $360^{\circ}$ span; with $m=8$ the same is fulfilled for finer step: $1.4^{\circ}$. Using common sense is no doubt necessary; but also experience turns out to be useful.

In this mechanism the initial value of each variable $g_{i}^{\circ}$ (to be defined in the input data) plays a role, since the values assumed by $g_{i}$ are $\left(k=1,2 \ldots 2^{m}\right)$

$$
g_{i}^{\circ}, \quad g_{i}^{\circ}-\Delta g_{i}, \quad g_{i}^{\circ}+\Delta g_{i}, \quad g_{i}^{\circ}-2 \Delta g_{i}, \quad g_{i}^{\circ}+2 \Delta g_{i}, \quad g_{i}^{\circ}-3 \Delta g_{i}, \ldots
$$

Note however that the $g_{i}^{\circ}$ are not critical quantities, provided they are internal to the span intervals.

\section{Creating the initial population. Constraints}

The first step of an evolutionary procedure is the creation of a population of tentative structures by means of a Montecarlo method (using e.g. pseudo-random numbers). ${ }^{9}$

One must assign the size of the population desired (e.g. 100-300 items) and the selection criteria (see below). Size and selection criteria control, in a very unforeseeable manner, the duration of the search, from few minutes to days. Anyway the search can be in any moment interrupted and restarted with new parameters, either creating a new list of solutions or queuing to the existing one.

If $K$ is the number of $g_{j}$ searched ( $K$ is of course well lower than $3 N$ ), the random search is performed generating, for each tentative structure, $K$ integer random numbers each in the range $0 \div 2^{m_{j}}-1$ to obtain a random combination of $g_{i}$. By using the cited subroutine LAGR, the corresponding model is created. It is evident that most of these models will be unfeasible. There are two alternatives: i) to retain all solutions, attaching to each solution a proper "penalty function", ii) to apply some selection criteria and reject immediately unfeasible items. The latter has been chosen in our procedure to avoid endless populations. Much experience is anyway necessary for calibrating the rejection parameters, actually under user control.

\subsection{Selection criteria. The xcon command}

The computer program implemented in our laboratory includes the following selection criteria (filters):

1) the 1st filter, most incisive and mandatory, is the one checking the connectivity of the trial structure versus the known connectivity (remember that the chemical formula is supposed known!). We define as connectivity a group of eight integer codes (connv) to be inserted

\footnotetext{
${ }^{9}$ True random numbers should be preferable. Some hardware devices are produced, but we do not have direct experience. Such devices should be of course very fast as billions random numbers are necessary.
} 
in the xcon command: the first is the number of atom-pairs separated by one bonds, the second the same number for two bonds, etc. up to 8 bonds. ${ }^{10}$

2) the 2nd filter eliminates structures with unfeasible bond angles. Non bonded pairs must have distances exceeding more than sepn 2 (atoms separated by two bonds) or sepn 3 (atoms separated by three or more bonds) the sum of covalent radii. Unrealistic structures with acute bond angles can so be eliminated. (In few cases, eg. cyclobutane, acute angles occur). The quantities sepn 2 and sepn 3 are supplied in the command xcon.

3) the 3rd filter checks the connections of the trial molecule with the neighbour (in molecular crystals no connections should be present; in linear polymer, instead, two connections take place); the number of external connections (linkno) is also assigned in xcon command. Connection are illegal if distance is lower than the sum of covalent radii augmented by tolnk, also supplied in xcon.

4) the 4th filter consists in computing the lattice energy, again rejection occurs if energy exceeds the assigned threshold, chosen by the user at the beginning of search.

5) the 5 th filter is based on the $R_{2}$ index defined as the residual $\chi^{2}$ (equation 2 ) divided by the sum of intensities. the tentative solution is rejected if $R_{2}$ exceeds an assigned value. Also for $R_{2}$ the threshold is chosen by the user.

Parameters sepn2, sepn3, tolnk, linkno and connv are defined in command xcon. Note that the same filters apply also in the subsequent breeding (see later).

It is evident the importance of assigning the above values cum grano salis, avoiding both excess or lack of severity. Indeed the ratio accepted-structures / generated-structures leans to be low (e.g. $10^{-5}$ ); the conformational freedom of the molecule has of course a critical role.

\subsection{Optimizing of the initial population}

We have introduced (as an optional) the local optimization of the random solutions found with the above procedure. Each random solution is a point in the (discontinuous) problem-space. The idea is to examine the nearest points in the problem-space and find points possibly more promising, based e.g. on $R_{2}$ index. In a K-dimension problem space points are $3^{K}-1$, a value prohibitively high if $K$ is large.

On belief that the local optimizazion is a good idea (experience seems to confirm) we have exploited a trick: to assign a reasonable value to the number of neighbouring points $P$ to be examined, say $500-1000$, and select, at random, $P$ points among the $3^{K}-1$ ones. ${ }^{11}$ With this contrivance the duration of an optimization step is reasonable.

\section{Genetic combinations}

The heart of the genetic procedure is the systematic combination (breeding) of the solutions belonging to the initial population following the rules of genetics. In this context two kinds of

${ }^{10}$ Establishing the connectivity codes is rather bothering, but the problem is easily resolved using TRY. One considers a dummy unit cell without symmetry and with large lattice constants. If the molecule is well constructed and displayed on the monitor the eight connection codes will appear on the top of the screen. Keep note and insert in xcon.

${ }^{11}$ For each integer $p$ running from 1 to $P$ one computes a real random number in the range $0-1$, multiply it by $3^{K}$ and truncate. The point to be considered is given by the digits of the product above written in the basis 3 . If desired 5 or 7 points can be considered instead 3 . 
combinations are considered: the cross-over and the mutation. A cross-over (binary combination) consists of an interchange of a selected gene belonging to the two mating items; a mutation (unary combination) is the change of a single bit (chosen at random) belonging to the bit-string. Considering a 9-gene example and a cross-over between genes $g_{2}, g_{7}$ one obtains:

Parent sequence (father):

Parent sequence (mother):

Child sequence:

Mutated sequence:

$\begin{array}{lllllllll}g_{1} & g_{2} & g_{3} & g_{4} & g_{5} & g_{6} & g_{7} & g_{8} & g_{9} \\ g_{1}^{\prime} & g_{2}^{\prime} & g_{3}^{\prime} & g_{4}^{\prime} & g_{5}^{\prime} & g_{6}^{\prime} & g_{7}^{\prime} & g_{8}^{\prime} & g_{9}^{\prime} \\ g_{1} & g_{7}^{\prime} & g_{3} & g_{4} & g_{5} & g_{6} & g_{2}^{\prime} & g_{8} & g_{9} \\ g_{1} & g_{7}^{\prime} & g_{3} & g_{4}^{*} & g_{5} & g_{6} & g_{2}^{\prime} & g_{8} & g_{9}\end{array}$

the symbol $*$ has been used for a mutation (the $g_{4}$ has been chosen by chance). In each case a new sequence is born for each crossover, two if also a mutation takes place. If the crossover takes place for all the pairs of $K$ genes, $K \times(K+1) / 2$ new strings occur; twice as many if also mutation is done.

Of course if two feasible structures are coupled, it is not warranted that child structures are meaningful. The above described "filtering" could be used again to eliminate unfeasible structures. At present the structures having $R_{2}$ higher than an assigned limit or a lattice energy higher than an assigned limit are eliminated. The limits, of course, should be fixed using experience. The new selected structures, anyway, are added to the initial ones.

The crossover can be done either considering a single gene, selected at random, or considering all genes; in the last case there are $K$ combinations, for each pair of mated structures. When the genetic combination is ended the population of solution is extended by an amount depending on the used filters. The new population will be again sorted using an appropriate figure like the $R_{2}$ index.

Examining the sorted list one observes, frequently, that the first solutions happen to be very similar to each other. That is no doubt an indication of success. If this is not the case a good idea is to repeat the breeding as many times as it is necessary. If however the breeding has resulted ineffectual in finding new solutions, it is better to repeat the whole process in more appropriate conditions. The exploration of a wide fraction of the problem-space is no doubt essential for the success.

\section{Procedure validation}

For testing purposes two already reported structures (single-crystal X-ray diffraction studies, molecular models are shown in Fig. 4) have been considered using published data. Only the reflections having $d>1.2 \AA$ have been considered to show that the method needs few reflections and that resolution can be modest.

The first test concerns the steroid equilin (a 20-atom molecule, ignoring hydrogen atoms) with restricted conformational freedom (Sawicki et al. 1999), the second test considers sucrose, a 23-atom molecule with high conformational freedom (Hynes et al., 1991). The latter was already considered in the first publication describing the method (Immirzi et al., 2008); the analysis has been repeated however in more challenging conditions. The input data for doing the two tests are given in Table 1 and Table 2; many comments are added for reader's convenience.

All computations were done using the program TRY (Immirzi, 2007) and assuming fixed bond-lengths in both cases, also fixed bond-angles in the case of equilin. The computing scheme is the simplest one: no hydrogen atoms, isotropic thermal vibration (an unique $B_{\text {iso }}$ ), unitary weight factors. In both cases the EA procedure consists of three phases: i) formation of 
the initial population, ii) improvement of solutions looking at adjacent points, iii) breeding by cross-over and mutation. The reliability of the resulting structures has been tested performing some cycles of full-matrix least-square refinement optimizing the same i.c. considered in the EA phase. The resulting $R$ indices are, of course, not competitive with the ones published because of the crude simplicity of the followed computation scheme and because of the reduced number of reflections. The LS convergency is anyway excellent.

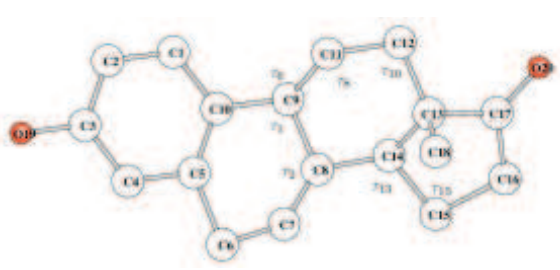

(a) Equilin

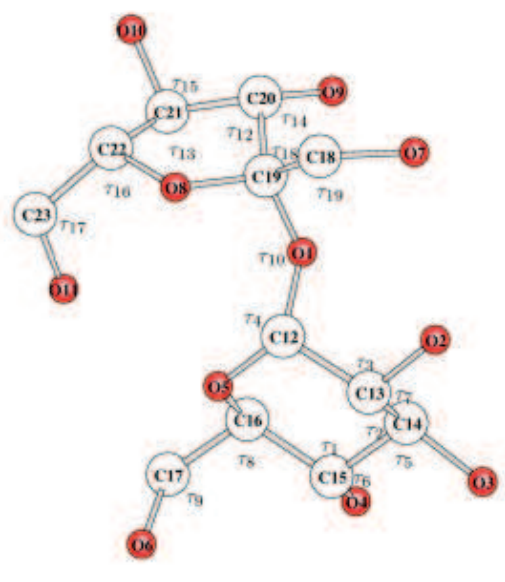

(b) Sucrose

Fig. 4. Molecular models of the two test molecules: equilin and sucrose. Bond angles $\left(\tau_{n}\right)$ are shown. Torsion angles $\left(\vartheta_{n}\right)$ are listed in Tables 1 and 3 .

The following scheme summarizes the essential data for the two tests.

$\begin{array}{lcc} & \text { equilin } & \text { sucrose } \\ \text { reflections considered }(d>1.2 \AA) & 715 & 589 \\ \text { number of non-hydrogen atoms } & 20 & 23 \\ \text { number of internal coordinates } & 60 & 68 \\ \text { varied internal coordinates } & 15 & 28 \\ \text { bits used in binary encoding } & 75 & 123 \\ \text { random structures generated } & 50 \times 10^{3} & 141 \times 10^{6} \\ \text { initial population } & 300 & 150 \\ \text { lowest } R_{2} \text { index after search } & 0.43 & 0.74 \\ \text { lowest packing energy } & -3 \mathrm{Kcal} & -11 \mathrm{Kcal} \\ \text { search duration } & 3 \mathrm{~min} . & 29 \mathrm{hr} . \\ \text { mated structures } & 20 & 30 \\ \text { resulting } R_{2} \text { after breeding } & 0.39 & 0.43\end{array}$

Note how the formation of initial population is performed in a very short time in the former case (75-bit encoding), and longer time in the latter (123 bit encoding). 


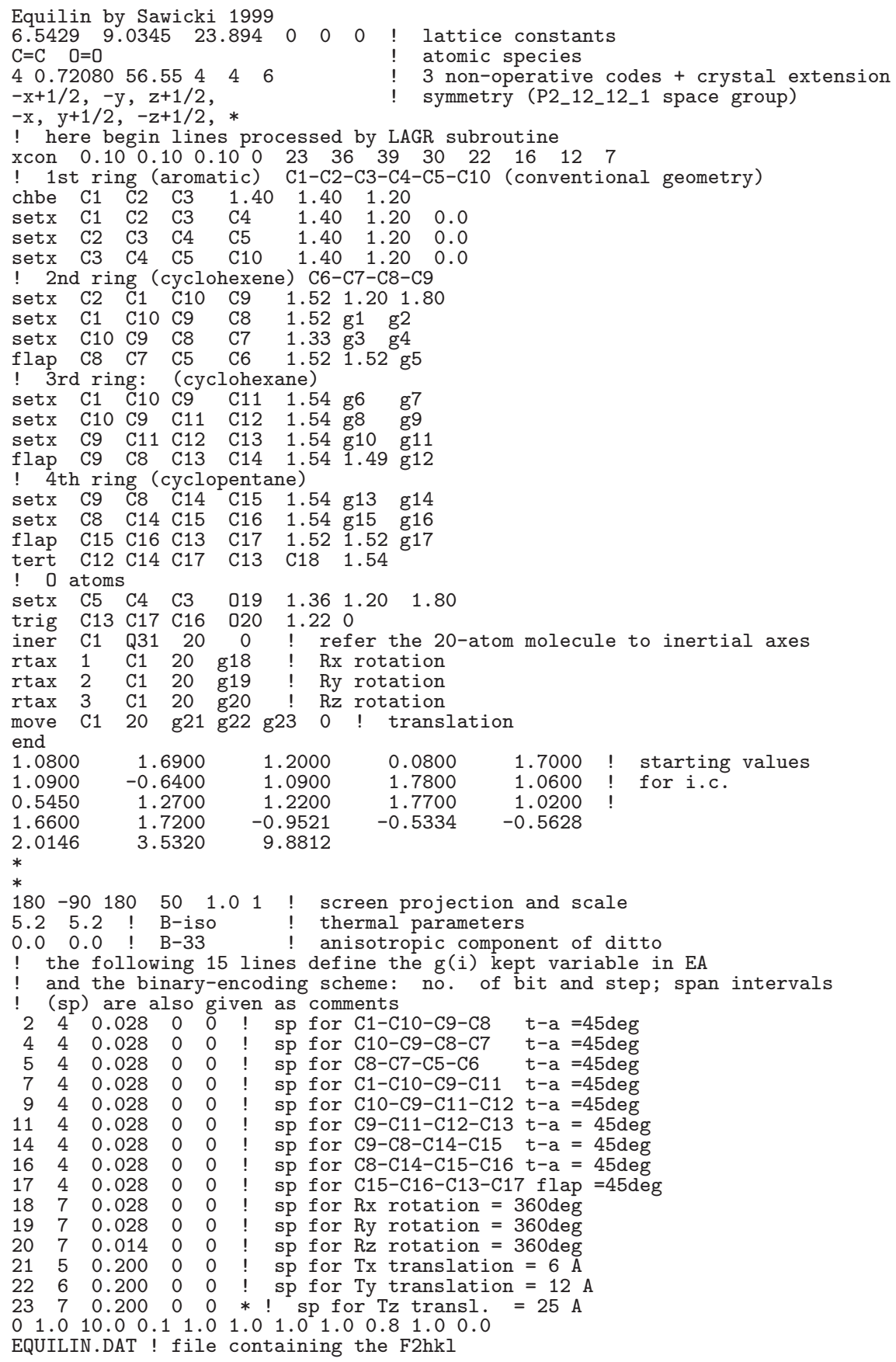

Table 1. Input data for equilin test 


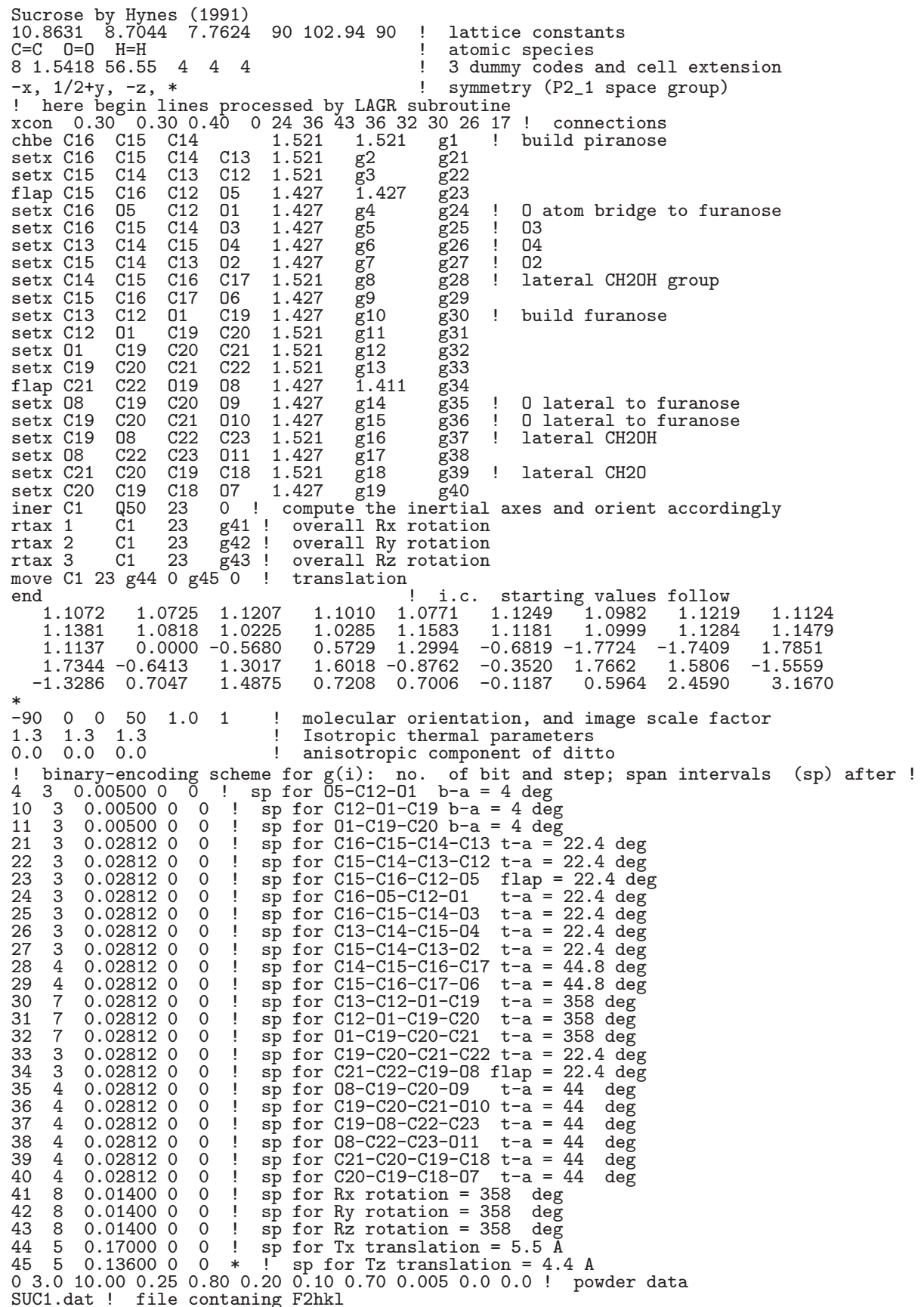

Table 2. Input data for sucrose test 


\section{Conclusions}

The power of evolutionary algorithms in resolving difficult crystal structures from diffraction data has been discussed evidencing the convenience of basing the approach on internal coordinates. A specific procedure has been implemented, having the following main features: i) it is based on internal coordinates (this reduces considerably the number of variables and their uncertainty); ii) it uses discretized coordinates and binary structure encoding (metaheuristic approach); iii) the procedure is designed as a constraint satisfaction problem so incorporating the numerous a priori information available; the constraints are considerd both in the initial step of the procedure (formation of the initial population of solutions based on Montecarlo methods) and in the subsequent steps (breeding of the population).

The ideas above afforded a Fortran-language computer program suitable for any kind of molecular structure and available free of charge. The evolutionary procedure has been inserted into a general-purpose program, entirely based on internal coordinates. The program, presently tested on known structures (in difficult conditions), runs well. It has however the disadvantage of needing numerous parameters (presently assigned by the user) which could be assigned automatically in more evolute versions of the program.

\section{References}

Arnott, S. \& Wonacott, A. J. (1966). Polymers, 7, 157-166

Back, T. (1996). Evolutionary Algorithms in Theory and Practice: Evolution Strategy, Evolutionary Programming, Genetic Algorithms, Oxford University Press, USA.

Bondi, A. J. (1964). J. Phys. Chem. 68, 441.

Cotton, A., Wilkinson G., Murillo, C.A., \& Bochmann, M. (1999). Advanced Inorganic Chemistry, 6th Ed. J. Wiley \& Sons, New York.

Davis, L (1991). Handbook of Genetic Algorithms, Van Nostrand Reinhold.

Eyring, H. (1932) Phys. Rev. 39, 746-748.

Feng, Z. J. \& Dong, C. (2006) J. Appl. Crystallogr., 39, 615-617.

Giacovazzo, C. (2002). Fundamentals of Crystallography, Oxford University Press, Oxford.

Goldberg, D. E. (1989). Genetic Algorithms in Search, Optimization and Machine Learning, Addison-Wesley, Berkeley.

Goldstein, H. (1980) Classical Mechanics 2nd Ed. Addison-Wesley, Berkeley.

Goto, H, \& Osawa, E. (1989) J. Am. Chem. Soc., 111, 8950-8951.

Hanson, A. J., Cheung, E. J. \& Harris, K. D. M. (2007) J. Phys. Chem. B, 111, 6349-6356.

Harris, K. D. M., Johnston, R. L. \& Kariuki, B. M. (1998) Acta Crystallogr., A54, 632-645.

Holland, J. (1975) Adaptation in Natural and Artificial Systems: an introductory analysis with applications to Biology, Control, and Artificial Intelligence, MIT press, USA.

Hynes, R. C., Le Page J. (1991). J. Appl. Cryst. 24, 352-354.

Ionita, M., Breaban, N., \& Croitoru C. (2010) Evolutionary Computation in Constraint Satisfaction in New Achievements in Evolutionary Computing, edt. P. Kovosec, Intech edition, Vukovar, Croatia.

Immirzi, A. (2007) (a). Chem. Inf. Model, 47, 2263-2265.

Immirzi, A. (2007) (b) J. Appl. Cryst., 40, 1044-1049

Immirzi, A., Erra, L., \& Tedesco, C. (2008). J. Appl. Cyrst. 41, 784-790.

Immirzi, A., L. Erra, \& Tedesco, C. (2009) J. Appl. Cryst., 42, 810-814.

Lagrange, J. L. (1797). Théorie des Fonctions Analytiques, Imprimerie de la République, Paris. 
Oganov, A. R., \& Glass, C. W. (2006). J. Chem. Phys., 124, art. 244704.

Okada, K., Noguchi, K., Okuyama, K., \& Arnott, S. (2003). Comput. Biol. Chem., 265-285

Polya, G. (1971). How to solve it. Princeton University Press.

Rietveld, H. M. (1967). Acta Crystallogr. 22, 151.

Rietveld, H. M. (1969). J. Appl. Crystallogr. 2, 65.

Sawicki, M. W., Li, N., \& Gosh. D. (1999). Acta Cryst. C, C55, 1999, 425-427.

Smith, P. J. C. \& Arnott, S. (1978). Acta Cryst., A34, 3-11.

Stout, G. H., \& Jensen, L. H. (1989). X-ray structure determination, a practical guide. J. Wiley \& Sons.

Tadokoro, H. (1979). Structure of Crystalline Polymers J. Wiley \& Sons, New York.

Weise, T. (2009). Evolutionary Algorithms. Theory and Application. URL : http://www.it-weise.de

Young, R. A. (1995) The Rietveld Method, IuCr Monograph on Crystallography, no. 5 


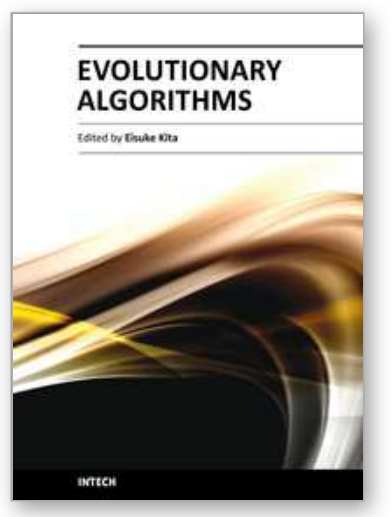

\author{
Evolutionary Algorithms \\ Edited by Prof. Eisuke Kita
}

ISBN 978-953-307-171-8

Hard cover, 584 pages

Publisher InTech

Published online 26, April, 2011

Published in print edition April, 2011

Evolutionary algorithms are successively applied to wide optimization problems in the engineering, marketing, operations research, and social science, such as include scheduling, genetics, material selection, structural design and so on. Apart from mathematical optimization problems, evolutionary algorithms have also been used as an experimental framework within biological evolution and natural selection in the field of artificial life.

\title{
How to reference
}

In order to correctly reference this scholarly work, feel free to copy and paste the following:

Attilio Immirzi, Consiglia Tedesco and Loredana Erra (2011). Evolutionary Algorithms in Crystal Structure Analysis, Evolutionary Algorithms, Prof. Eisuke Kita (Ed.), ISBN: 978-953-307-171-8, InTech, Available from: http://www.intechopen.com/books/evolutionary-algorithms/evolutionary-algorithms-in-crystal-structure-analysis

\section{INTECH}

open science | open minds

\section{InTech Europe}

University Campus STeP Ri

Slavka Krautzeka 83/A

51000 Rijeka, Croatia

Phone: +385 (51) 770447

Fax: +385 (51) 686166

www.intechopen.com

\section{InTech China}

Unit 405, Office Block, Hotel Equatorial Shanghai

No.65, Yan An Road (West), Shanghai, 200040, China

中国上海市延安西路65号上海国际贵都大饭店办公楼405单元

Phone: +86-21-62489820

Fax: $+86-21-62489821$ 
(C) 2011 The Author(s). Licensee IntechOpen. This chapter is distributed under the terms of the Creative Commons Attribution-NonCommercialShareAlike-3.0 License, which permits use, distribution and reproduction for non-commercial purposes, provided the original is properly cited and derivative works building on this content are distributed under the same license. 